\title{
COVID-19 and The Conundrum About How to Return to School for Children with Epilepsy
}

\author{
Geetanjali S Rathore* \\ Department of Pediatrics, University of Nebraska Medical Center, Omaha, Nebraska, United States
}

*Corresponding author: Geetanjali S Rathore, Department of Pediatrics, University of Nebraska Medical Center, Omaha, Nebraska, United States

\begin{abstract}
The global pandemic of coronavirus disease 2019 (COVID-19) is caused by severe acute respiratory syndrome coronavirus 2 (SARS-CoV-2) and was first identified in December 2019 in Wuhan, China. Limited data suggests children are low risk and get less severe COVID-19 infection. As the outbreak of COVID-19 continues to spread, children with special health care needs may be at increased risk for complications. The forced school closure early in the year may have allowed for social distancing and protected children at risk from exposure to the virus. As the schools prepare to open in person schooling, the parents and caregivers of children with epilepsy are faced with unchartered and challenging decisions about how their child will return to school. When deciding between in-person and virtual learning, limited data is available for parents and health care providers to make this decision for children with epilepsy. Presence of other comorbidities may also alter the decision and needs for school. A Parent is their child's best advocate, who knows them and their needs the best. Some of the important considerations that can help make the best-informed decision for this special population are discussed in this communication.
\end{abstract}

Keywords: Epilepsy; Disabilities; Rescue Medications; Guidelines; COVID-19; Virtual Learning; School

Abbreviations: COVID -19 (Coronavirus disease 2019); SARS-CoV-2 (Severe acute respiratory syndrome coronavirus 2); CDC (Centers for disease control and prevention); AAP (American Academy of Pediatrics); ACTH (Adrenocorticotropic hormone); UNMC (University of Nebraska Medical Center); IEP (Individualized Education Program); IT (Information technology)

\section{Introduction}

Coronavirus disease 2019 (COVID-19) is a novel infectious disease caused by severe acute respiratory syndrome coronavirus 2 (SARS-CoV-2) which initially occurred in Wuhan, China in late 2019. This virus has now become a pandemic rapidly spreading globally [1]. While children have been reported to be less affected by COVID-19 compared to adults, children with certain medical conditions may be at increased risk for severe illness [1]. Children who are medically complex, who have serious genetic, neurologic, metabolic disorders, and with congenital heart disease might be at increased risk for severe illness from COVID-19. One centers for disease control and prevention (CDC) report noted that the majority of hospitalized children with COVID-19 in the United States had one or more underlying medical conditions such as chronic lung disease, cardiovascular disease, and immunosuppression [2]. Epilepsy is a neurological disorder characterized by a spontaneous

recurrence of unprovoked seizures. It is one of the most common chronic neurological conditions, with a reported prevalence rate of $0.7-1.0 \%$ with high incidences in elderly people and children [3]. With the limited data available, it is unknown if patients with epilepsy have a higher risk for infection or severe complications of COVID-19. Various medical associations and societies for patients with epilepsy suggest that the history of epilepsy in itself seems unlikely to be a risk factor for COVID-19 [4]. Epilepsy, most authors will argue, does not consist only of epileptic seizures, but also many systemic co-morbidities with a common origin [5]. The CDC has indicated that due to high rate of associated comorbidities may increase the risk for COVID-19 infection in patients with epilepsy [4]. Conversely, children with well-controlled epilepsy and no other comorbidities may remain asymptomatic or exhibit only mild symptoms of COVID-19 [6]. 
Early in the year, in response to the COVID-19 pandemic, hundreds of countries implemented school closures worldwide [7]. This kept vast majority of children, including those with complex medical conditions, at home and allowed to maintain social distancing. With the schools gearing to re-open, parents of children with epilepsy have a daunting burden of uncertainty and fear of the unknown risk. The CDC, the American Academy of Pediatrics (AAP), and most local health and education departments are recommending re-opening of school in the United States. According to the CDC, children, and adolescents under 18 years old account for less than 7 percent of COVID-19 cases and less than 0.1 percent of COVID-19-related deaths in the United states [8]. Studies suggest that COVID-19 transmission among children in schools may be low. International studies, where schools have re-opened, have assessed how readily COVID-19 spreads in schools. They are reporting low rates of transmission when the community transmission is low. Current data from these also shows the rate of infection among younger school children, and from students to teachers, has been low, especially if proper precautions are followed. There have also been few reports of children being the primary source of COVID-19 transmission among family members [9-11]. This is consistent with data from both virus and antibody testing, suggesting that children are not the primary drivers of COVID-19 spread in schools or in the community [12-14]. The results are still not conclusive, but the available evidence points to the theory that in-person schooling is in the best interest of students, particularly in the context of appropriate mitigation measures being implemented. This low presumed risk for children is countered by the potential harm prolonged school closure may cause in the long term. It can lead to significant learning loss, particularly in students with heightened behavioral and special needs for whom in-person instruction is vital $[15,16]$. Of another particular concern is the disparities in educational outcomes caused by school closures for low-income and minority students and students with disabilities [16].

What does this mean for children with epilepsy and their families? Parents, caregivers, and guardians of children with epilepsy face new and challenging decisions about how their child will return to school this fall, such as deciding between inperson and virtual learning. As mentioned above, limited data about COVID-19 in children suggest that children are less likely to get COVID-19 than adults, and when they do get COVID-19, they generally have less serious illness than adults. However, it is clearly identified that children who are medically complex, who have neurologic, genetic, metabolic conditions, or who have congenital heart disease might be at increased risk for severe illness from COVID-19, compared to other children. Severe illness means that they may require hospitalization, intensive care, or a ventilator to help them breathe, or may even die [2]. Children with epilepsy qualify for more than one of the listed risk factors due to use of immunosuppressive medications, being non ambulatory, respiratory weakness/insufficiency, intellectual disability, and mental health/behavioral disorders. CDC has provided a 'Decision making' tool for parents to try to make a calculated decision about in person versus distance learning for their child [17]. The questions in these tools are designed to help you weigh the risks and benefits of available educational options before you make decisions. This tool is a general tool and may not completely apply to children who are higher risk due to epilepsy and its co-morbidities but give some idea of what broadly needs to be considered.

Some of the modified questions listed below may still be helpful for families to think about and try to come to an informed decision about return to school for their child with epilepsy.

a) Does my child have an underlying condition that increases the risk for severe illness from COVID-19? Children with epilepsy with well controlled seizures and no other comorbidities would not be considered very high risk based on the known literature. Certain underlying health factors may put children with epilepsy at higher risk from COVID-19, regardless of seizure control. Intellectual disability or behavior problems may lead to poor compliance with wearing a mask, following guidelines placed for social distancing, hand hygiene and other risk mitigation strategies. Most seizure medications do not lead to immune suppression, but a small proportion of epilepsy patients are on Adrenocorticotropic hormone (ACTH), steroids, Everolimus or immunomodulating therapies. These special drugs may reduce the immunity of epilepsy patients and place them at higher risk for severe infection with COVID-19. Having poor swallowing, aspiration, weak pulmonary clearance, obesity, heart disease would all place patients with epilepsy in the high-risk category based on CDC guidelines [18].

b) Is my child's caregiver or anyone else living at home, at increased risk for severe illness from COVID-19 due to age or underlying medical conditions? If the child with epilepsy is not high risk based on CDC criteria [18], make sure their caregivers/ family members are not at high risk due to an underlying comorbidity, If you do identify someone at home at risk it might be a consideration for avoiding in person school for the child to protect the other family members.

c) What is the level of community spread in my local area? A critical role for the safe and successful opening of schools is a low local infection rate in the community. According to the University of Nebraska Medical Center (UNMC) Public health department and biocontainment center, the 15 countries internationally were successful in reopening in person schools because they waited until transmission rates were less than 10 cases/million population/day [19]. The ability or timing to return to in person schooling will also factor in the local health authority's preparedness with testing, timely contact tracing and quarantine. The best protection for the children, teachers, and school staff from getting COVID-19 infection is reducing the rates of transmission in the community. 
d) What is my school's plan for COVID preparedness? Do I feel comfortable with it? Ask your local school or school district for what their reopening plans. Make sure you clearly understand and feel comfortable with the steps they are taking to reduce the spread of COVID-19. Know how your school is planning to implement social distancing, hand washing, mask wearing, frequent disinfection and proper ventilation. Consider if your child will be able to follow the guidelines effectively. If your child will be need modifications, discuss with your school how they will keep your child safe and still support their educational goals.

e) Does your school have plans for taking care of children with special needs? Check with the school and the school nurse what is their proposed plan to take care of children with special needs following COVID-19 prevention guidelines. If the school nurse gets sick, is there a backup, especially if your child with epilepsy needs any medication or medical care during school hours. If a child needs an aid or para, ensure that proper screening, masking and social distancing can be maintained. Discuss any medications, including rescue medications that may need to be administered at school. Will these be still available safely and in a timely manner if the child has an emergency. Make sure you have an updated seizure action plan and if any special considerations are needed, have them listed as a part of this plan.

f) Is my child's mode of transportation to and from school safe? If your child uses public transportation for school, know what measures are going to be taken to reduce the risk of spreading COVID-19 during transportation. Some proposed measures by the medical organizations are- decreased bus/ transit capacity, mandatory mask wearing, increased cleaning, and disinfecting practices. If your child needs a wheelchair or other special equipment, be informed about how they will accommodate these measures for your child. Another important consideration is administration of emergency care/ rescue medications during transportation and planning to do so safely maintaining COVID-19 precautions.

g) Is Virtual/At-Home Learning Feasible for my child and our family? To make sure your child succeeds in their educational goals, assess if virtual learning will be feasible for your home environment. Ensure that you or someone you have identified will be able to supervise virtual learning for your child at home. Another aspect to consider would be if the distance learning is compatible with the learning style and needs of your child. Access to reliable internet and digital device for your child to use would also be needed. Some schools may provide digital devices and IT (Information technology) support for home. If planning on doing virtual learning for your child, have a detailed Individualized Education Program (IEP) outlines with input not only from teachers and special needs education specialists but also the IT personnel.

h) Does my child receive school-based services? If your child received physical therapy, occupational therapy, speech therapy, mental health services or other similar school-based services, will you still be able to receive these services virtually or have an at home option? If your school provides services only on site and your child is high risk, address these concerns with your school. A major proportion of children with epilepsy have an individualized education plan, specialized classes, or extra tutoring. Assessing if your child will have access to these services virtually/at home and will they meet your child's needs adequately is a critical factor to ensure your child thrives in their academic and social goals.

\section{Conclusion}

In conclusion, deciding whether or not to send your child back to school in person during the COVID-19 pandemic can be a very difficult decision for parents of children with epilepsy and other co-morbidities. The safety data for children returning to in person school is extremely limited at the current time. The health organizations are guiding local school districts to make the best efforts to try to continuously evaluate the situation and plan a safe return to school for our children. These measures are however mostly tailored for children who are presumed not to be at high risk for COVID-19. There are conditions in children identified at high risk by the CDC, but no clear guidelines are available for these families or schools on how to make their return to school safely. To make matters more confounded, if epilepsy is high risk or not may depend on other comorbidities or therapies the child may be on. When weighing decisions about your child returning to school, it is important to consider your child's/family's unique needs, the school's ability to accommodate these safely at school or virtually at home, and the situation of the infection rates of COVID-19 in your local community. A vital part of the decision making will be consulting with your health-care providers, local health care authorities and school representatives regarding your concerns, special considerations or safety measures pertaining to the COVID-19 risk mitigation for your child.

\section{References}

1. Wan S, Xiang Y, Fang W, Yu Zheng, Boqun Li, et al. (2020) Clinical features and treatment of COVID-19 patients in northeast Chongqing. J Med Virol 92(7): 797-806.

2. CDC COVID-19 Respsonse Team (2020) Coronavirus disease 2019 in Children-United States, MMWR Morb Mortal Wkly Rep 69(4): 422-426.

3. Fiest KM, Sauro KM, Wiebe S, Patten SB, Kwon CS, et al. (2017) Prevalence and incidence of epilepsy: a systematic review and meta-analysis of international studies. Neurology 88(3): 296-303.

4. Naoto Kuroda (2020) Epilepsy and COVID-19: Associations and important considerations. Epilepsy and Behavior 108. 
5. Alan WC Yuen, Mark R Keezer, Josemir W Sander (2018) Epilepsy is a neurological and a systemic disorder. Review, Epilepsy and Behavior 78 57-61.

6. Lu Q, Shi Y (2020) Coronavirus disease (COVID-19) and neonate: what neonatologist need to know. J Med Virol 92(6): 564-567.

7. The Lancet Child Adolescent Health (2020) Pandemic school closures Risks and opportunities. Lancet Child Adolesc Health 4(5): Pp 341.

8. (2020) United States COVID-19 Cases and Deaths by State. CDC, Georgia.

9. (2020) COVID-19 in schools-the experience in NSW. National-Centre for-immunization-research-and-surveillance (NCIRS), NSW, Australia.

10. Ludvigsson JF (2019) Children are unlikely to be the main drivers of the COVID-19 pandemic - A systematic review. Acta Paediatr 109(8): 15251530 .

11. Kostas Danis, Olivier Epaulard, Thomas Bénet, Alexandre Gaymard, Séphora Campoy, et al. Cluster of coronavirus disease 2019 (Covid-19) in the French Alps, 2020. Clinical infectious diseases 71(15): 825-832.

12. World Health Organization (WHO) (2020) Report of the WHO-China Joint Mission on Coronavirus Disease 2019 (COVID-19). Pp: 1-40.
13. (2020) Children and COVID-19. National Institute for Public Health and the Environment, Ministry of Health, Welfare and Sport, Netherlands.

14.Gudbjartsson DF, Helgason A, Jonsson H, Olafur T Magnusson, Pall Melsted, et al. (2020) Spread of SARS-CoV-2 in the Icelandic Population. N Engl J Med 382(24): 2302-2315.

15. Dorn E, Hancock B, Sarakatsannis J, Viruleg E (2020) COVID-19 and student learning in the United States: the hurt could last a lifetime. McKinsey\&Company Pp: 1-9.

16. Gross Bethany, Alice Opalka (2020) Too Many Schools leave Learning to Chance During the Pandemic. Center for Reinventing Public Education (CRPE), USA.

17. (2020) Back to School Decision Making Tool. US Centers for Disease Control and Prevention, CDC, USA.

18. (2020) US Centers for Disease Control and Prevention, CDC, USA.

19. John Lowe, Jocelyn Herstein, Brandon Grimm, David Brett Major, Ali S Khan, et al. (2020) COVID-19 Back to School Play Book: Guiding Principles to Keep Students, Teachers, and Staff Safe in K-12 Schools. Pp: 1-36.
To Submit Your Article Click Here:

This work is licensed under Creative Commons Attribution 4.0 License

Submit Article

DOI: $10.32474 / 0 J N B D .2020 .04 .000184$

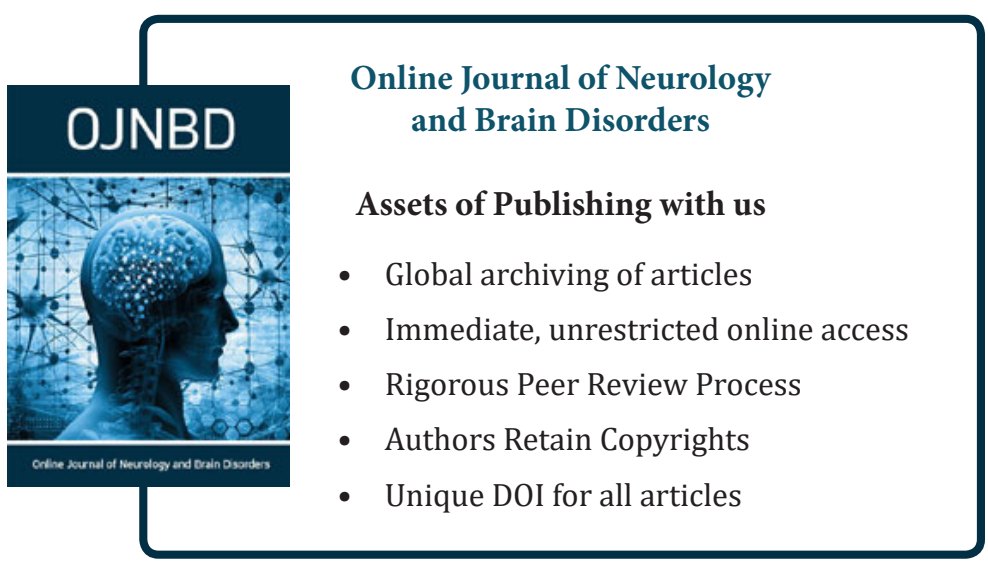

